\begin{tabular}{|c|c|c|c|}
\hline Chan,2016(BD) & - & $1.26(0.87,1.85)$ & 4.54 \\
\hline Hernandez,2016(LD) & $\rightarrow$ & $1.51(1.25,1.82)$ & 11.61 \\
\hline Hernandez,2016(HD) & $\rightarrow$ & $1.32(1.17,1.50)$ & 24.60 \\
\hline Graham,2016(LD) -major ECH & 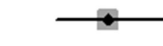 & $1.58(1.32,1.90)$ & 11.29 \\
\hline Graham,2016(HD) & - & $1.48(1.32,1.67)$ & 22.98 \\
\hline Lip,2016(BD) & & $1.05(0.74,1.49)$ & 7.34 \\
\hline Noseworthy,2016(BD) & - & $1.30(1.10,1.53)$ & 17.65 \\
\hline $\begin{array}{l}\text { Heterogeneity: } \mathrm{Tau}^{2}=0.0055, \mathrm{Chi}^{2}=8.12, \\
\mathrm{df}=6,(\mathrm{p}=0.229) ;\left.\right|^{2}=26.1 \% \\
\text { Test for overall effect: } \mathrm{z}=24.87(\mathrm{p}<0.0001)\end{array}$ & & $1.38(1.27,1.49)$ & 100.00 \\
\hline
\end{tabular}

Abstract 45 Figure 2 Rivaroxaban compared with Dabigatran in risk of major bleeding in AF patients. AF, atrial fibrillation. ECH, extracranial hemorrhage.

than warfarin for the prevention of IS/TE in AF patients. Major bleeding risk was significantly higher with rivaroxaban than dabigatran, as was all-cause mortality and GIB. Rivaroxaban was comparable to warfarin for major bleeding, with an increased risk in GIB and decreased risk of ICB.

\section{AUDIT OF NEW ORAL ANTICOAGULANT MONITORING IN PRIMARY CARE; ARE PATIENTS BEING PRESCRIBED THE CORRECT DOSE?}

Anik Ghai* , lan Duffus. Western Sussex Hospital NHS trust

\subsection{6/heartjnl-2017-311726.45}

Non-vitamin K antagonist oral anticoagulants (NOACs) are increasingly being used for the prophylaxis of stroke in patients with non-valvular atrial fibrillation (NVAF). One of their limitations is their reliance on renal clearance making them potentially more unsafe in patients with renal impairment. NICE recommend regular renal function testing in these patients as well as drug dose adjustments to be made according to their calculated $\mathrm{CrCl}$.

Aims 1. To identify patients with NVAF who have been prescribed NOACs and to measure the percentage of those that have had renal function tests done in the last 12 months.

2. To determine if patients are being prescribed the correct anticoagulant dose as per their $\mathrm{CrCl}$ ?

3. Are $100 \%$ of patients having their renal function and weight monitored at the correct frequency according to NICE recommendations?

Methods Data was collected from two GP practices in Bognor Regis. $\mathrm{CrCl}$ was calculated for each patient by using the Cockgroft-Gault equation. The dose of anticoagulant was then reviewed for each patient to see if it was appropriate as per their $\mathrm{CrCl}$.

Results 176 patients were identified. 45\% of patients were prescribed Apixiban, 41\% were prescribed Rivaroxaban and $14 \%$ of patients prescribed Dabigatran.

The average age of patients was 77 years old with a median age group of 78 years.

$84.5 \%$ of patients on NOACs had their renal function tested in the last 12 months with the remainder of patients having their renal function monitored at varying time periods greater than 12 months and less than 3 years. It was found that one patient had no record of their renal function.

In order to calculate their $\mathrm{CrCl}$, weight measurement is also required. However only $49 \%$ of patients had their weights checked in the last 12 months. $4 \%$ of patients had no recorded weights.

Accurate CrCls could only be measured in 46\% (81/176) of patients, of whom had both renal function and weights recorded in the last 12 months.

Assuming stable weights, all patients prescribed apixiban and dabigatran were prescribed the correct dose as per their $\mathrm{CrCl}$, however only $81 \%(59 / 73)$ of patients taking rivaroxban were on the correct dose. 2 patients were under anticoagulated despite normal $\mathrm{CrCl}$, and 12 patients despite having impaired renal function $(\mathrm{CrCl}<50)$ were prescribed the higher dose. Patients with impaired renal function $(\mathrm{CrCl}<60)$ did not have their renal function monitored more frequently as is suggested by NICE.

Summary Whilst the majority of patients have had their renal function tested in the last 12 months, a significant number are not having their weight checked. Accurate dosing of the NOACs therefore cannot be done as creatinine clearances are not being accurately measured. Incorrect dosing increases risks of adverse events such as bleeding. Findings from this study were presented at the two GP surgeries during practice meetings to raise awareness of the problem and incorrect doses were rectified.

\section{A WIDE RANGE OF CATHETER ABLATIONS CAN BE SAFELY PERFORMED WITHOUT INTERRUPTING NOVEL ORAL ANTICOAGULANTS}

${ }^{1}$ Selvakumar Velu*, 'Janaka Pathiraja, ${ }^{1}$ Andrew Lapper, 'Geoff Kidd, ${ }^{1}$ Anita Arya, ${ }^{2}$ Craig Barr, ${ }^{2}$ Peter Forsey, 'Sanjiv Petkar. 'New Cross Hospital; ${ }^{2}$ Dudley Group NHS foundation trust

\subsection{6/heartjnl-2017-311726.46}

Introduction Catheter ablation, in the immediate peri-and post procedural period is associated with a transiently increased thrombogenic state secondary to atrial inflammation arising as a result of endothelial damage caused by manipulation of ablation catheters and/or ablation lesions. For atrial fibrillation(AF) ablations, the 2016 European Society of cardiology Guidelines recommend uninterrupted warfarin therapy in the peri- 
procedural period. In observational cohorts and one randomised study (PIONEER-AF), uninterrupted NOAC therapy has also been found to be safe in patients undergoing AF ablations. EHRA states that simple ablation procedures have a low bleeding risk while complex ablation procedures have high bleeding risk. We describe our experience of a wide variety of catheter ablations performed on uninterrupted NOAC therapy and compare our results with similar procedures undertaken on uninterrupted warfarin therapy.

Methods A retrospective analysis of the NICOR database for all ablations undertaken on uninterrupted anticoagulation at the New Cross Hospital, Wolverhampton, between April 2014 and August 2016 was undertaken. Data regarding the number and type of procedures, type of oral anticoagulation (warfarin versus NOACs), frequency of trans-septal punctures, DCCV during procedures and complications were analysed.

Results Atrial of 648 ablations were performed in the study period of which328(50.7\%) were undertaken on uninterrupted anticoagulants (uninterrupted warfarin group (uW Grp): 228 (35.1\%) and uninterrupted NOAC group (UNOAC Grp): 101 (15.5\%). Mean age was 59 in both groups with more male preponderance in the NOAC group. A range of simple and complex ablations were done including 131 (57.4\%) AF in UW Grp compared to $26(26.5 \%)$ in the uNOAC grp. Transseptal punctures were more common in the UW Grp compared to the uNOAC Grp, however cardioversions (both external and internal) were used in similar fashion. Composite of bleeding and thrombo-embolic complications were relatively low in both the groups $[n=5(2.1 \%)$ in the VKA group compared to $n=1(0.9 \%)$ in the UNOAC Grp with OR 2.21 (95\% CI 0.25 to $19.2 ; \mathrm{p}=0.47)]$.

Conclusions Our experience suggests that a wide range of simple and complex ablation procedures can be safely performed ion uninterrupted NOAC therapy. Complications in the uNOAC Grp were lower than the uW Grp, reflecting growing confidence among electrophysiologists to undertake such procedures even in the absence of antidote for majority of the NOACs.

\begin{tabular}{lll} 
Abstract & $\mathbf{4 7}$ & Table 1 \\
\hline Variables & $\begin{array}{l}\text { Uninterrupted Warfarin group } \\
(\mathbf{n}=228)\end{array}$ & $\begin{array}{l}\text { uninterrupted NOAC group } \\
(\mathbf{n}=101)\end{array}$ \\
\hline Mean Age & 59 & 59 \\
Male & $59 \%$ & $67 \%$ \\
Mean & 2.02 & 2.1 \\
CHA2DS2VAsc & & \\
Simple ablations & 90 & 76 \\
Complex & 139 & 27 \\
ablations & & \\
Trans septals & 149 & 27 \\
DCCV & 67 & 23 \\
Complications & 10 & 2 \\
\hline
\end{tabular}

\section{UTILISATION OF CARDIAC IMPLANTABLE ELECTRONIC DEVICE THERAPY IN PATIENTSWITH ATTR-WILD TYPE AMYLOIDOSIS WITH CONCURRENT CARDIAC INVOLVEMENT}

Anwar A Chahal* ${ }^{*}$ Deepak Padmanabhan, Nidhi Tandon, Virend K Somers, Angela Dispenzieri, Martha Grogan, Peter A Brady, Grace Lin. Mayo Clinic

\subsection{6/heartjnl-2017-311726.47}

Introduction The role of cardiac implantable electronic device (CIED) therapy in patients with ATTR-wt (senile) amyloid is not very well defined. It is unknown whether there is mortality benefit or harm with the implantation of CIEDs.

Methods We performed a retrospective analysis of a prospectively collected registry of patients with biopsy proven ATTRwt amyloid with cardiac involvement. These patients were followed up at Mayo Clinic, Rochester, Minnesota between Jan 1, 1985 to Sep 30, 2015. The follow-up of these patients was done via personal communication (written or verbal) and entered into the database. During analysis these patients were subdivided on the basis of the presence or absence of a CIED, cardiac involvement or not, and all-cause mortality analysed. The implantation of a CIED was analysed as a timedependent covariate in the survival of these patients and Kaplan-Meier (K-M) survival plots created. Comparison between groups with CIED and those without CIED was made. In addition, CIEDs were subdivided into permanent pacemakers (PPM), defibrillators (ICDs) and cardiac resynchronization devices (CRT-P/D). Mortality among the patients with each device subtype was estimated and compared to overall mortality.

Results 409 patients with ATTR-wt and cardiac involvement were reviewed with CIEDs implanted in 101 (25\%) patients. The median follow-up (25th, 75th centile) was 2.4 (1.1, 4.3) years. Utilisation of ICDs in this population was higher than the general community in Olmsted county. There was no association in the overall survival or evidence of harm with CIED utilisation in patients with ATTR amyloidosis and cardiac involvement.

Conclusions CIEDs utilisation remains high in ATTR-wt with cardiac involvement. Due diligence is needed in selecting the optimal patient for benefit prior to the implantation of CIEDs in this cohort. Further research is required to pool multicentre amyloid registry data and determine if there is mortality benefit.

\section{PREDICTING RISK OF SCD IN FABRY DISEASE: A SINGLE CENTRE EXPERIENCE}

${ }^{1}$ Shanat Baig*, ${ }^{1}$ Nicky Edwards, ${ }^{2}$ Sabrina Nordin, ${ }^{1}$ Boyang Liu, ${ }^{2}$ James Moon, ${ }^{1}$ Tarek Geberhiwot, ${ }^{1}$ Richard P Steeds. 'Queen Elizabeth Hospital; ${ }^{2}$ Barts Heart Centre

10.1136/heartjnl-2017-311726.48

Introduction Fabry disease (FD) is a rare X-linked lysosomal storage disorder with a variable cardiac phenotype and a 